\title{
Widespread headache as the first clinical manifestation of giant cell arteritis in patients affected by polymyalgia rheumatica
}

\section{Ciro Manzo}

Internal and Geriatric Department ASL NA 3 sud, Rheumatologic Outpatient Clinic hospital “Mariano Lauro”, Sant'Agnello, Italy

\begin{abstract}
Introduction: In giant cell arteritis (GCA) headache of new onset due to inflammatory involvement of the temporal artery (TA) represents a diagnostic criterion. A widespread headache (WH) with scalp tenderness due to cranial arteritis can represent another manifestation of GCA.

Material and methods: In 225 elderly patients with polymyalgia rheumatica (PMR) followed in our rheumatologic outpatient clinic from 2004 until June 2016, the frequency of WH as the first clinical manifestation of GCA was evaluated.

Results: Among 26 patients with GCA+PMR (11.6\% of total) , 5 (23.07\%) had WH as first clinical manifestation of GCA without TA. In all these patients TA colour duplex sonography (CDS) and 18-fluorodeoxyglucose positron emission tomography (FDG-PET) with total body contrast-enhanced CT was consistent with the diagnosis of arteritis. TA biopsy was not performed. High doses of prednisone ( $1 \mathrm{mg} / \mathrm{kg} /$ day) led to the immediate and total disappearance of the headache.

Conclusions: The widespread headache should be considered as the first symptom GCA and in cases of suspicion of vasculitis patients should have a full diagnostics examination. Colour duplex sonography and FDG-PET with total body contrast-enhanced CT are useful tools for non-invasive diagnosis of GCA.
\end{abstract}

Key words: polymyalgia rheumatica, giant cell arteritis, tension headache, widespread headache.

\section{Introduction}

Giant cell arteritis (GCA) is considered one of the most common vasculitides in older persons [1]. The branches of the external carotid (including the temporal arteries) are typically involved but every medium- and large-size vessel can be involved. $40-60 \%$ of GCA patients have also manifestations of polymyalgia rheumatica (PMR) whereas 10-16\% of PMR patients can have manifestations of GCA [2]. Headache of new onset is the most frequent clinical manifestation and represents a diagnostic criterion [3]. Typically it is due to inflammatory involvement of temporal artery (TA), is associated with TA tenderness and decreased pulsation and with a significant increase of inflammatory markers (erythro- cyte sedimentation rate- ESR; C-reactive protein - CRP). A widespread headache $(\mathrm{WH})$ with scalp tenderness due to cranial arteritis can represent another manifestation of GCA: when it is associated with TA (as usually), there are no diagnostic difficulties; but when it is not and the inflammatory markers are in the normal ranges, differential diagnosis with tension-type headache (TTH) (a type of headache with different therapy and prognosis) is very difficult. As TA, WH can be associated with other typical GCA manifestations such as visual loss (due to ischemia in the territory of the ophthalmic artery) and/or jaw claudication (due to insufficient blood flow to the masseter and pterygoid muscles). Therefore its recognition is essential for the adjustment of corticosteroid therapy. 


\section{Material and methods}

We followed 225 elderly patients with PMR in our rheumatologic outpatient clinic from 2004 to June 2016. In PMR+GCA patients we evaluated the frequency of WH as first clinical manifestation of GCA. In all patients with PMR and WH, a TA clinical examination, a TA colour duplex sonography (TA-CDS), the determination of ESR and CRP serum levels, a fundus oculi examination and a 18-fluorodeoxyglucose positron emission tomography (FDG-PET) with total body contrast-enhanced computerized tomography (CT) were performed. TA-CDS was performed by the same ultrasonographer, who was unaware of the patient's clinical data. TA-CDS was carried out after a variable time period (from 2 to 4 months) after the onset of headache: the presence of a headache not controlled by analgesics and present even at night was the main warning, even without inflammatory markers raised. FDG-PET was performed by the same diagnostic center (outside our hospital); it was considered positive for diagnosis in the presence of vascular glucose uptake greater than liver uptake. TA biopsy was not performed.

\section{Results}

Among 26 patients (18 females; 8 males, median age $=$ 76 years) with GCA+PMR (11.6\% of total), 5 (23.07\%) had $\mathrm{WH}$ as first clinical manifestation of GCA without TA and without elevation of ESR and CRP. In all these patients TA CDS showed a halo sign (Fig. 1) and all had a FDG-PET picture consistent with the diagnosis of arteritis. The characteristics of these patients are reported in Table I.

When we compared PMR-GCA/WH group (5 patients) vs. PMR-GCA/TA group (21 patients), some significant differences were highlighted: the average time for a correct diagnosis was statistically significant (3 months as average vs. 2 weeks); the onset of vision loss was more frequent in the second group in which the vision loss was the first clinical GCA manifestation in two patients; ESR and CRP were (obviously) much higher in TA group (Table II). No other vascular symptoms occurred in our patients. The switch of corticosteroid dosages from 10/12.5 mg pred-

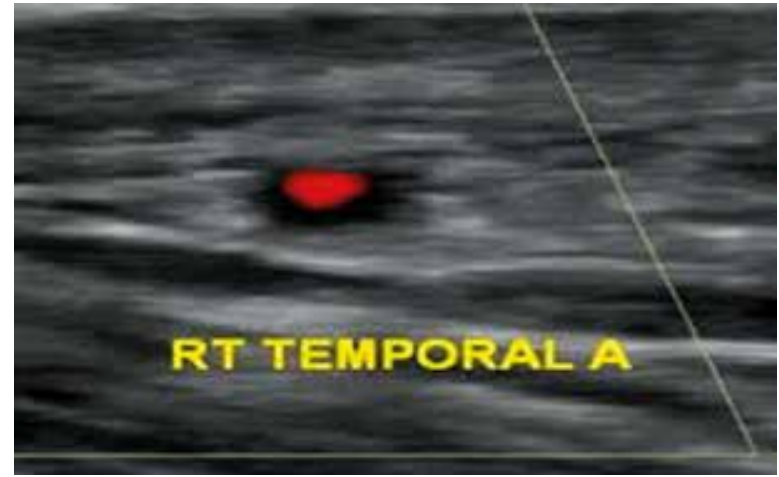

Fig. 1. HALO-SIGN in temporal artery colour duplex sonography.

Table I. Baseline characteristics of the five patients

\begin{tabular}{|cc|}
\hline Gender, $n$ (\%) & $\begin{array}{c}\text { Male - 1 (20\%) } \\
\text { Female -4 (80\%) })\end{array}$ \\
\hline $\begin{array}{c}\text { Age at the date of examination, } \\
\text { average (min-max) [years] }\end{array}$ & 73 (68-84) \\
\hline ESR (min-max) $[\mathrm{mm} / \mathrm{h}]$ & $22-30$ \\
\hline CRP (min-max) $[\mathrm{mg} / \mathrm{l}]$ & $2.2-6.00$ \\
\hline $\begin{array}{c}\text { Disease duration before the } \\
\text { diagnosis of WH, min-max [months] }\end{array}$ & $2-8$ \\
\hline Patients with halo sign in TA CDS (\%) & 100 \\
\hline Patients with FDG-CT positive (\%) & 100 \\
\hline
\end{tabular}

$E S R$ - erythrocyte sedimentation rate; $C R P$ - C-reactive protein; TA CDS - temporal artery colour duplex sonography; FGD-CT 18fluorodeoxyglucose positron emission tomography with total body contrast-enhanced computerized tomography

nisone or equivalent/day (as average) to $1 \mathrm{mg} / \mathrm{kg} /$ day allowed a prompt and permanent resolution of $\mathrm{WH}$.

\section{Discussion}

Rarely WH can be the initial manifestations of GCA in PMR patients, in absence of TA. The possibility that GCA can present without elevation of inflammatory markers is well known [4-6]: up to $25 \%$ of biopsy-confirmed GCA

Table II. Patients with WH [5] vs. patients with classical TA [21] as first clinical manifestation of GCA overlapping PMR

\begin{tabular}{|cccc|}
\hline Parameter & WH & TA & $p$ value $^{*}$ \\
\hline Gender, $n(\mathrm{M}, \mathrm{F})$ & $1 \mathrm{M}, 4 \mathrm{~F}$ & $7 \mathrm{M}, 14 \mathrm{~F}$ & $<0.000$ \\
\hline Age at the date of examination - min-max [years] & $68-84$ & $70-85$ & NS \\
\hline ESR - min-max $[\mathrm{mm} / \mathrm{h}]$ & $22-30$ & $68-110$ & $<0.0000$ \\
\hline CRP - min-max $[\mathrm{mg} / \mathrm{l}]$ & $2.2-6.00$ & $12-66.4$ & $<0.0000$ \\
\hline Disease duration before diagnosis, min-max [months] & $2-8$ & $0.5-1.00$ & $<0.005$ \\
\hline Visual loss, $n$ & 0 & 2 & $<0.000$ \\
\hline
\end{tabular}

\footnotetext{
${ }^{*}$ Fisher exact test. $P$ value was considered significative when $<0.05$.
} 
patients present with a normal ESR and CRP before corticosteroids are started [7, 8].

TA biopsy is one of the diagnostic criteria for GCA $[3,9]$ but it is to consider that the absence of typical biopsy features does not exclude diagnosis of GCA [5]. In 1995 Schmidt et al. first described using Doppler ultrasonography a 'halo' sign [10] that is a hypoechoic halo in the artery wall probably caused by oedema. In a meta-analysis published in 2010, Ball et al. [11] highlighted that in the presence of the halo sign the probability of a positive biopsy is high (sensitivity $=75 \%$; specificity $=$ $83 \%$ ). Besides, the presence of halo sign was even more specific when measured against the ACR criteria instead of TA biopsy (sensitivity $=69 \%$; specificity $=89 \%$ ) [11] TA-CDS has been proposed as a useful alternative tool when temporal biopsy cannot be performed [12-14]. A complementary contrast-enhanced $\mathrm{CT}$ was acquired immediately after FDG. The metabolic information obtained from 18F-FDG PET combined with the demonstration of wall enhancement and thickness in cranial and extracranial vessels assessed by contrast-enhanced CT is considered more useful for diagnosis of GCA [15]. In other words, in our five patients the absence of TA biopsy did not exclude the diagnosis of GCA since other important clinical and instrumental elements were present.

When WH is present without raised inflammatory markers, TTH must be considered in the differential diagnosis [16]. TTH is known to have a very different therapy and prognosis. The presence of WH during the night and an absence of efficacy of drugs commonly used for TTH should represent important warnings. In our experience, WH (when is a GCA manifestation) is always associated with subclinical involvement of other medium and large-size vessels but no vascular symptom was clinically observed when diagnosis of GCA was made. The very small size of our cohort is a limitation. Therefore the data presented deserve to be confirmed on data from multicenter cohorts. Nevertheless, in consideration of the close relationship between GCA and PMR, WH of recent onset - even if not associated with raised inflammatory markers in PMR patient - should be considered as a possible manifestation of overlapping GCA and specific diagnostic investigations implemented $[17,18]$.

$$
* * *
$$

The possibility that $\mathrm{WH}$ without TA involvement can be a favorable prognostic factor for the onset of vision loss should be evaluated in broader statistics.

\section{Acknowledgements}

The author thanks Dr. Maria Natale and Dr. Raffaele Cioffi for their valuable collaboration when they worked in the rheumatologic outpatient clinic of hospital "Mariano Lauro"; Dr. Giuseppe Fiorentino, who conducted all TA
CDS; Dr. Giacomo Visco, chief of Neurological Outpatient Clinic of "Mariano Lauro" for some helpful tips on TTS.

The author declare no conflict of interest.

\section{References}

1. Weyand CM, Goronzy JJ. Medium- and large-vessel vasculitis. N Engl J Med 2003; 349: 160-169.

2. Salvarani C, Cantini F, Hunder GG. Polymyalgia rheumatica and giant-cell arteritis. Lancet 2008; 372: 234-245.

3. Hunder GG, Bloch DA, Michel BA, et al. The American College of Rheumatology 1990 criteria for the classification of giant cell arteritis. Arthritis Rheum 1990 ; 33: 1122-1128.

4. Kermani TA, Schmidt J, Crowson CS, et al. Utility of erythrocyte sedimentation rate and C-reactive protein for the diagnosis of giant cell arteritis. Semin Arthritis Rheum 2012; 41: 866-871.

5. Helliwell T, Muller S, Hider S. ESR can be normal in giant cell arteritis and polymyalgia rheumatica. BMJ 2012; 344: e1408.

6. Martínez-Taboada VM, Blanco R, Armona J, et al. Giant cell arteritis with an erythrocyte sedimentation rate lower than 50 . Clin Rheumatol 2000; 19: 73-75.

7. Muratore F, Cavazza A, Boiardi L, et al. Histopathologic findings of patients with biopsy-negative giant cell arteritis compared to those without arteritis: a population-based study. Arthritis Care Res (Hoboken) 2016; 68: 865-870.

8. Laria A, Zoli A, Bocci M, et al. Systematic review of the literature and a case report informing biopsy-proven giant cell arteritis (GCA) with normal C-reactive protein. Clin Rheumatol 2012; 31: 1389-1393.

9. Mukhtyar C, Guillevin L, Cid MC, et al. EULAR recommendations for the management of large vessel vasculitis. Ann Rheum Dis 2009; 68: 318-323.

10. Schmidt WA, Kraft HE, V̈olker L, et al. Colour Doppler sonography to diagnose temporal arteritis. Lancet 1995; 345: 8669.

11. Ball AL, Walsh SR, Tang TY, et al. Role of ultrasonography in the diagnosis of temporal arteritis. Br J Surg 2010; 97: 1765-1771.

12. Schmidt WA, Seifert A, Gromnica-Ihle E, et al. Ultrasound of proximal upper extremity arteries to increase the diagnostic yield in large-vessel giant cell arteritis. Rheumatology 2008; 47: 96-101.

13. Diamantopoulos AP, Haugeberg G, Lindland A, Myklebust G. The fast-track ultrasound clinic for early diagnosis of giant cell arteritis significantly reduces permanent visual impairment: towards a more effective strategy to improve clinical outcome in giant cell arteritis? Rheumatology (Oxford) 2016; 55: 66-70.

14. Schmidt WA. Ultrasound in vasculitis. Clin Exp Rheumatol 2014 ; 32 (1 Suppl 80): S71-S77.

15. Henes JC, Müller M, Krieger J, et al. [18F] FDG-PET/CT as a new and sensitive imaging method for the diagnosis of large-vessel vasculitis. Clin Exp Rheum 2008; 26 suppl 49: S47-S52.

16. Headache classification committee of the International Headache Society (IHS). Cephalalgia 2013; 33: 629-808.

17. Rana $A Q$, Saeed U, Khan $O A$, et al. Giant cell arteritis or tension-type headache? A differential diagnostic dilemma. J Neurosci Rural Pract 2014; 5: 409-411.

18. Ruiz M, Pedraza MI, de la Cruz C, et al. Headache in the elderly: characteristics in a series of 262 patients. Neurologia 2014; 29: 321-326. 